\title{
Correlations between facial emotion recognition and cognitive flexibility in autism spectrum disorder
}

\author{
Rosa Angela Fabio, Sonia Esposito, Cristina Carrozza, Gaetana Pino and Tindara Caprì
}

\begin{abstract}
Purpose - Various studies have examined the role of executive functions in autism, but there is a lack of research in the current literature on cognitive flexibility in autism spectrum disorders (ASD). The purpose of this study is to investigate whether cognitive flexibility deficits could be related to facial emotion recognition deficits in ASD.
\end{abstract}

Design/methodology/approach - In total, 20 children with ASD and 20 typically developing children, matched for intelligence quotient and gender, were examined both in facial emotion recognition tasks and in cognitive flexibility tasks through the dimensional change card sorting task.

Findings - Despite cognitive flexibility not being a core deficit in ASD, impaired cognitive flexibility is evident in the present research. Results show that cognitive flexibility is related to facial emotion recognition and support the hypothesis of an executive specific deficit in children with autism.

Research limitations/implications - One of the limit is the use of just one cognitive test to measure cognitive flexibility and facial recognition. This could be important to be taken into account in the new research. By increasing the number of common variables assessing cognitive flexibility, this will allow for a better comparison between studies to characterize impairment in cognitive flexibility in ASD.

Practical implications - Investigating impairment in cognitive flexibility may help to plan training intervention based on the induction of flexibility.

Social implications - If the authors implement cognitive flexibility people with ASD can have also an effect on their social behavior and overcome the typical and repetitive behaviors that are the hallmark of ASD.

Originality/value - The originality is to relate cognitive flexibility deficits to facial emotion.

Keywords Identification, Autism spectrum disorder, Child, Cognitive flexibility, Autism, Facial emotion recognition

Paper type Research paper

\section{Introduction}

Many individuals with autism spectrum disorder (ASD) are characterized by impairments in social interaction such as difficulty in forming friendships and a lack of understanding emotions and feelings of others (Kaland et al., 2007). They also show impairments in facial emotion recognition (APA, 2013). The ability to discern emotions from facial expressions is essential to join in social interaction, and the reverse may be true as well: social interaction, by increasing one's experience with expressive faces, may be necessary for the typical development of facial emotion recognition.

Research has demonstrated that children with ASD show an atypical processing of emotions, in particular in recognition, interpretation and expression of emotions. The most known explanation is a deficit in theory of mind, that is the ability to represent mental states of oneself and others to understand behaviors (Castelli et al., 2013; Frye et al., 1995; Kaland et al., 2007; Leo et al., 2019; Marchetti et al., 2020).
(Information about the authors can be found at the end of this article.)

Received 22 February 2019 Revised 11 February 2020 Accepted 21 February 2020

(c) Rosa Angela Fabio, Sonia Esposito, Cristina Carrozza, Gaetana Pino and Tindara Caprì. Published by Emerald Publishing Limited. This article is published under the Creative Commons Attribution (CC BY 4.0) licence. Anyone may reproduce, distribute, translate and create derivative works of this article (for both commercial and non-commercial purposes), subject to full attribution to the original publication and authors. The full terms of this licence may be seen at http://creativecommons.org/ licences/by/4.0/legalcode

Conflict of interest: The authors declare no conflict of interest and no funding. All participants and their parents gave written informed consent. The American Psychological Association's ethical standards were met in the conduct of this study and the Human Ethics Committee of Cognitive Science, Psychological, Educational and Cultural Studies of the University of Messina approved the study protocol. 
Another explanation is the weak central coherence hypothesis. Individuals on the autism spectrum tend to be more, sometimes overly, focused on details; this can often be at the expense of understanding the actual meaning or appreciating the nature of a situation or context (Happe and Frith, 2006).

With reference to the social impairment of subjects with ASD, looking at the face holds particular significance and provides information, which is important for communication and survival. As seen above, children with ASD process faces differently and show reduced attention to faces and facial expressions (Dawson et al., 2004b; Klin et al., 2002). They show deficits in face recognition (Weigelt et al., 2013). It is unclear if the reduced orienting to faces is the result or the cause of the theory of mind deficit (Baron-Cohen et al., 2013; Dawson et al., 2010). Children with ASD may also not find others' facial expressions intrinsically rewarding. This aspect characterizes the lack of interest in faces thus impairing their facial processing skills so that children with ASD do not become "face experts" like their typically developing peers (Dawson et al., 2005).

Children with ASD also show deficits in cognitive flexibility (Russell, 1997). Cognitive flexibility refers to the ability to shift to different thoughts or actions depending on situational demands (Van Eylen et al., 2011). It has been shown that flexibility deficits in ASD are related to repetitive behaviors in ASD. Inflexible adherence to specific routines, resistance to change, difficulty in transitions between different locations or events or circumscribed interests and inordinate preoccupation with particular objects or activities have commonly been observed in individuals with ASD (Baron-Cohen et al., 2013; Carrozza and Fabio, 2020; Fabio, 2012; Fabio et al., 2011). However, there is a lack of research in current literature on cognitive flexibility in ASD and the results of the few studies are conflicting. Some studies showed that children with ASD and healthy controls had no differences in terms of cognitive flexibility (Happé et al., 2006). In contrast, other studies found marked differences (Yerys et al., 2012). As argued by De Vries and Geurts (2012), the inconsistency of the results presented in literature on cognitive flexibility in ASD might be because of the heterogeneity of the cognitive profiles in ASD, high levels of comorbidity and overlap of different executive functions (Kenworthy et al., 2008; De Vries and Geurts, 2012; Fabio et al., 2016; 2018a; 2018b; 2018c; 2018d; Fabio and Caprì, 2019a; Fabio et al., 2019b; 2019c). Conill et al. (2014) demonstrated a link between cognitive flexibility and facial processing skills in children with ASD, especially when different contextual cues are present to extract facial emotion.

From the weak central coherence hypothesis, individuals with autism tend to be overly focused on details and this can lead to deficit in the attention process, and consequently, in an executive process such as the cognitive flexibility; in the same way, this over-focus can also affect facial emotion recognition that needs both analytic capacity and recognition of the global gestalt.

The main aim of this study was to explore whether cognitive flexibility deficits could be linked with facial emotion recognition deficits in ASD. First, the performance of children with ASD and typically developing subjects (TD), matched for intelligence quotient (IQ) and gender, was examined in a task related to facial emotion recognition of different emotions. Second, cognitive flexibility through dimensional change card sorting (DCCS) with two types of stimuli (faces and trucks) was assessed. Finally, correlations between cognitive flexibility and facial recognition of different emotions in both groups were investigated. In accordance with previous studies (Russell, 1997; Conill et al., 2014), a cognitive flexibility deficit in children with ASD compared to TD children and a correlation between cognitive flexibility and face recognition were expected.

\section{Methods \\ Participants}

The ASD group included 20 children ( 14 men and 6 women) between 38 to 45 months ( $M=$ 42.93, SD 2.53). All received a formal diagnosis of ASD made by a multidisciplinary team, according to diagnostic and statistical manual of mental disorders, fifth edition criteria (APA, 
2013). The children were recruited through the University Hospital "G. Martino" Messina, division of child neurology and psychiatry.

To select children with ASD, the parental interview ADI-R (autism diagnostic interview - revised) and autism diagnostic observation schedule (ADOS; Lord et al., 2000) were used. Cognitive and language development was assessed at each age using the Griffiths mental development scales (Griffiths, 1984). None of the ASD children had a known neurological or genetic disorder.

The ASD group was individually matched for mental age and verbal IQ with a control group of 20 toddlers ( $M=29.37, S D=3.66$ ) with typical development. These children were recruited from a public school and none of them presented any neurological or psychiatric disorders or had a first-degree family member with a developmental, learning or neurological disorder. None of these children were on medication. This information was gathered during a telephonic interview with the parents. The characteristics of the participants are summarized in Table I.

\section{Performance measures}

Dimensional change card sorting task. To examine cognitive flexibility, both a standard and a modified version of dimensional change card sorting task (DCCST) were used (Zelazo and Palfai, 1995). The DCCST is a task that measures cognitive flexible switching between dimensions. In both versions, children are asked to sort cards varying in two dimensions (usually shape and color) into two boxes marked by target cards, each of which matches exactly one dimension.

In the first phase, pre-switch, children are asked to sort by one dimension (e.g. shape). In the second phase, post-switch, children are asked to switch to sorting the same cards by a different dimension (e.g. color). Children's ability to sort correctly in the post-switch phase is taken as a measure of children's cognitive flexibility ability.

In the standard version, the stimuli cards were trucks and stars, whereas, in the modified version they were the faces of men and women. The materials consisted of laminated cards $(9 \times 11 \mathrm{~cm})$ with a white background. The bottom wall of each sorting tray is $28 \times 13 \mathrm{~cm}$ and the base is $13 \times 11 \mathrm{~cm}$. In both versions, the stimuli were $12 \mathrm{cards}$, each containing a figure with a specific shape (truck or star; and male or female) and color (blue or red for truck and star; and yellow or green t-shirt for male or female) on a white background. Each card was mounted on a vertical wall of a sorting tray (Figures 1 and 2).

DCCT procedure. Children were randomly assigned to sort one of two sets of picture cards into trays. In the pre-switch phase, children were asked to sort 12 cards by one dimension; on the post-switch phase, children were asked to switch and sort the same 12 cards by the other dimension (Figure 1). The first sorting rule (color or shape in the standard version; and color or gender in the modified version) was randomized across children.

\section{Table I Characteristics of the groups}

\begin{tabular}{lcc} 
Characteristics & $\begin{array}{c}\text { ASD group }(\mathrm{n}=20) \\
M(S D)\end{array}$ & $\begin{array}{c}\text { Control group }(\mathrm{n}=20) \\
M(S D)\end{array}$ \\
\hline No. of boys/girls & $6 / 14$ & $6 / 14$ \\
Age in months & $42.93(2.53)$ & $29.37(3.66)$ \\
IQ tot. Griffiths & $79.08(2.53)$ & $83.75(4.06)$ \\
Locomotor subscale & $21.07(3.03)$ & $27.29(4.79)$ \\
Personal social subscale & $19.33(3.93)$ & $25.91(4.58)$ \\
Hand and eye coordination subscale & $8.69(1.36)$ & $21.04(3.40)$ \\
Hearing and speech subscale & $19.10(4.15)$ & $26.37(2.92)$ \\
Performance subscale & $20.92(4.18)$ & $21.16(4.14)$ \\
ADOS-T total & $16.00(5.00)$ & $3.30(2.4)$ \\
Mental age on ADOS scores & 28.7 & 29.2
\end{tabular}




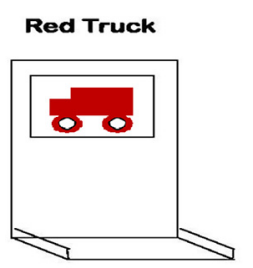

Blue Star

The Cards to be Sorted

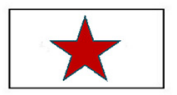

Red Star
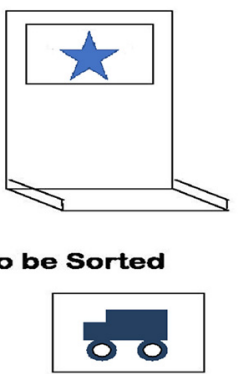

Blue Truck

Figure 2 Sorting boxes with model cards affixed

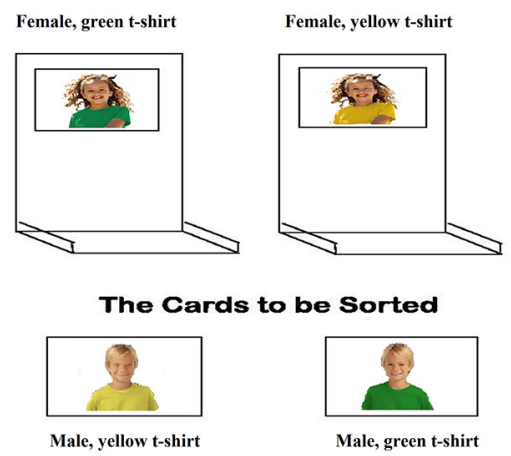

The instructions on pre-switch trials followed this form: "in this game, we're going to sort these cards by shape. So, in this game, all the trucks go in this box with this truck, and all the stars go in this box with this star." On post-switch, the need to switch was made explicit, following this form: "now we're going to change the game; we're not going to sort by shape anymore. Now we're going to sort the cards by color. So, in this game, all the red ones (trucks and stars) go in this box with this red card and all the blue ones go in this box with this blue card." Every trial throughout the task began with an explicit statement of the sorting rule (e.g. "all the red ones go in here with this red card, and all the blue ones go in here with this blue card"), and when a sorting card was presented to the child, both dimensions were labeled (e.g. "here's a red truck. Where does it go?"). The cards were placed face down in the tray as they were sorted. The order of sorting cards was randomized with the constraint that the same card did not appear more than twice in succession. Corrective feedback was only given during the pre-switch phase.

Facial emotion recognition. For facial emotion recognition, all faces were recruited from the standardized battery. The task was divided into two phases. In the first phase (recognition task), participants were told to observe six face items followed by six car items and to remember them as best as they could. All items were presented sequentially at center of screen for $3 \mathrm{~s}$ each. The task started with a fixation cross on the screen. The face items represented six emotions: happiness, sadness, surprise, fear, disgust and anger (Figure 3).

In the second phase (short-memory task), participants were told to remember the previous items. Six face pairs followed by six car pairs were presented on the screen. The pair of items were shown simultaneously side-by-side until the participant responded, which one was "old" 
(seen previously), guessing if necessary. Each pair consisted of one old and one new/ distractor item. The old item was on the right 50 per cent of the time. The order of all items was the same in each phase of the task. The correct number of responses was the parameter.

\section{Procedure}

All participants with ASD were tested individually in a quiet room. They were tested in two sessions. Before the test phase, facial and color processing skills were assessed through some figures of female and male people with colored t-shirts. They completed the facial discrimination task and DCCS. The control subjects were tested in the school where they were recruited. The order of these tasks was counterbalanced to avoid order effects.

\section{Statistical analyses}

Data were analyzed using SPSS Version 14.0 for Windows. The descriptive statistics of the dependent variables were tabulated and examined. Alpha level was set to 0.05 for all statistical tests. In the case of significant effects, the effect size of the test was reported. The Bonferroni correction was applied for multiple comparisons.

\section{Results}

The cognitive flexibility parameter was coded through the number of correct responses (CR) according to the shift of the rule (shape, color and face) in four phases of the test. A multivariate analysis of variance with repeated measures 2 (groups: ASD vs TD) $\times 2$ (type of stimuli: truck vs star) $\times 2$ (phases: before shift vs after shift) was used. The type of stimulus

Figure 3 Items of the facial emotion recognition task
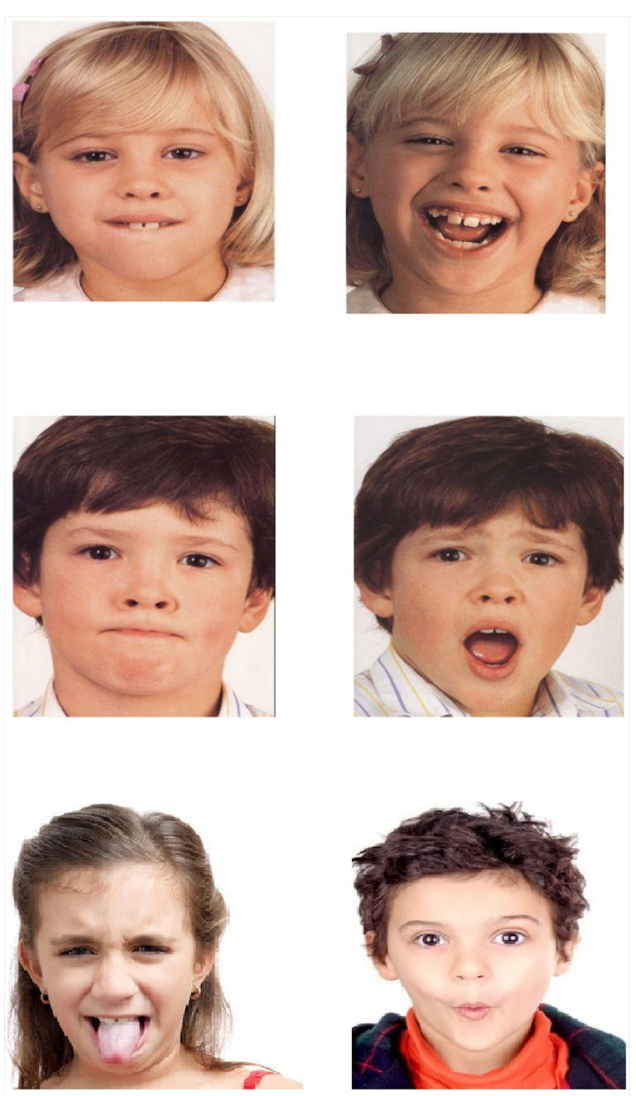
factor showed significant effect, $F(1,38)=325.29, p<0.0001$ and $d=0.9$. The group factor also showed significant effect, $F(1,38)=14.17, p<0.05$ and $d=0.78$; this indicated that the performance of ASD group was lower than the control group.

With reference to the phases factor, there was also a significant effect, $F(1,38)=14.17, p<$ 0.0001 and $d=0$ 0.9. The interaction Groups $X$ phases was also significant, $F(1,38)=$ 15.52, $p<0.001$ and $d=0.9$; after the shift, the performance was lower for ASD $t(19)=2.23$, $p<0.05$, while the control group showed a similar performance before and after the shift $t$ (19) $=0.23$ and $p=0.61$.

Table II shows the means and standard deviations related to CR in facial emotion recognition in the ASD and the control groups.

Group factor showed significant effect with reference to facial emotion recognition, $F(1$, $38)=568.27, p<0.0001$ and $d=0.9$. This result indicated that subjects with typical development showed performances that reached the ceiling effect while the ASD group had lower performances.

With reference to the correlations between the facial emotion recognition task and cognitive flexibility, results showed that the increase of the cognitive flexibility indices was correlated to a better capacity to recognize an emotional face, $r(39)=0.41$ and $p<0.03$.

\section{Discussion}

The purpose of the current study was to investigate whether cognitive flexibility could be linked with facial emotion recognition in children with ASD, compared with typically developing children. The results showed that the ASD group consistently perceives emotional facial expressions differently from typical children, confirming the emotional impairments in ASD. In addition, with reference to DCCST, we found significant differences between the ASD group and the control group. Precisely, both groups had lower performance in DCCST when the stimuli were the faces, but the performance of the ASD group was even lower than the control group.

Our results are in line with previous studies (Russell, 1997) and confirm that children with ASD have cognitive flexibility impairment. However, our findings are in disagreement with the results of De Vries and Geurts (2012) as these authors found a cognitive flexibility deficit only in a subgroup of children with ASD, measuring cognitive flexibility through a gender emotion switch task. Probably, this difference could be related to the type of task used in both experiments and the heterogeneity of the cognitive profiles in ASD.

An interesting finding of the current study was that cognitive flexibility was correlated to facial recognition. As expected, this confirms the presence of a correlation between cognitive flexibility and facial recognition. Although this correlation seems consistent, in the current study, it has proven difficult to clearly articulate the links between the two factors. We think that the weak central coherence hypothesis can better explain these results. The reduced capability of subjects with ASD in attention because of over-selectivity leads to both deficits in executive processes and facial emotion recognition. In other words, it is possible that children with ASD have an impaired cognitive flexibility and this may bring about difficulties in facial emotional recognizing.

\section{Table II Means and SD of CR in facial discrimination tasks}

\begin{tabular}{lcc} 
Tasks & $\begin{array}{c}\text { ASD group } \\
M(S D)\end{array}$ & $\begin{array}{c}\text { Control group } \\
M(S D)\end{array}$ \\
\hline Recognition task & & \\
Male faces & $2.75(0.86)$ & $6.00(0)$ \\
Female faces & $2.33(0.88)$ & $5.83(0.38)$ \\
Short term memory task & $1.33(1.15)$ & $5.83(0.38)$
\end{tabular}


The face recognition is an important index in the formation of social cognition and neurodevelopment in humans. Corrigan's model proposed four cognitive abilities as prerequisites for adequate social behavior and cognition, such as emotion recognition, understanding of social situations and other people's intentions and cognitive flexibility. In light of the results of the present study, applying this model to children with ASD suggests that the facial recognition deficit could due to failures to adjust one's behavior in accordance with the demands of the situation, that is, in flexibility. Impairments in cognitive flexibility could mean that children with ASD are unable to recognize facial expressions.

Beyond cognitive flexibility, a possible explanation for the facial recognition deficit may be that children with ASD have problems in the neurocognitive mechanisms that represent facial expression information and/or multi-modal expression information (Loth et al., 2018). Other recent observations would seem to suggest that facial recognition deficits in ASD may be linked to the high frequency of comorbidity with alexithymia (Bird and Cook, 2013; Cook et al., 2013). However, the debate on factors related to facial recognition impairments in ASD is still open, because the studies on facial expression recognition produced mixed results, consequently there can be different possible explanations for this deficit and potential future directions.

Future studies should examine the link between cognitive flexibility and facial recognition skills in other neurodevelopmental disorders. Instead of just including a typically developing control group, comparison between individuals with ASD and other neurodevelopmental disorders can elucidate the difficulties of individuals with ASD. Moreover, other cognitive functions, for example, attention, can be potential underlying mechanisms to facial recognition deficits in ASD. Thus, future research could investigate the relationship between facial recognition and a broad range of cognitive functions within individuals with ASD.

A limit of the present study is that the sample size is small, so caution is needed to generalize the findings of this study to the ASD population as a whole. Larger sample may have resulted in more significant correlations, consequently future studies with a larger samples are needed to obtain reliable results from groups.

Given that the mixed results can be because of the sensitivity of the test used in the previous research, the use of other cognitive tests to measure cognitive flexibility and facial recognition could be an additional point to be taken into account in new research. By increasing the number of common variables assessing cognitive flexibility, this will allow for a better comparison between studies to characterize impairment in cognitive flexibility in ASD. Investigating impairment in cognitive flexibility related to other neuropsychological parameters in children with ASD has important clinical implications in terms of understanding the repetitive and restricted behavior that is the hallmark of ASD.

The present study has some clinical implications. Facial expression recognition is critical for many aspects of social communication and daily life, consequently, it becomes important to understand the nature of this deficit in the ASD population to develop specific rehabilitative training. Unfortunately, the need for treatment and support does not end as children with ASD become older, it is usually necessary throughout life. For all these reasons, determining the integrity of facial recognition in ASD is essential to teaching social skills and improve the quality of life for patients and caregivers.

\section{References}

American Psychiatric Association (APA) (2013), Diagnostic and Statistical Manual of Mental Disorders, 5th ed. American Psychiatric Publishing, Arlington, VA.

Baron-Cohen, S., Tager-Flusberg, H. and Lombardo, M.V. (Eds) (2013), Understanding Other Minds: Perspectives from Social Cognitive Neuroscience, 3rd ed., Oxford University Press, Oxford, ISBN 978-019-852446-5.

Bird, G. and Cook, R. (2013), "Mixed emotions: the contribution of alexithymia to the emotional symptoms of autism", Translational Psychiatry, Vol. 3 No. 7, pp. e285-e285. 
Carrozza, C. and Fabio, R.A. (2020), "Dysfunctional perceptual antecedent can justify the social orienting deficit in autism spectrum disorder: an eye-tracking study", Autism Research, Vol. 1

Castelli, I., Antonietti, A., Fabio, R.A., Lucchini, B. and Marchetti, A. (2013), "Do Rett syndrome persons possess theory of mind? Some evidence from non-treated girls", Life Span and Disability XVI, Vol. 2, pp. 157-168.

Conill, E., Stilgenbauer, J., L., Mouren, M., C. and Gousse, ", V. (2014), "Rôle de la flexibilité cognitive dans la reconnaissance d'expressions émotionnelles chez les personnes atteintes de troubles du spectre autistique", Annales Mé dico-Psychologiques, Vol. 172 No. 5, pp. 392-395.

Cook, R., Brewer, R., Shah, P. and Bird, G. (2013), "Alexithymia, not autism, predicts poor recognition of emotional facial expressions", Psychological Science, Vol. 24 No. 5, pp. 723-732.

Dawson, G., Webb, S.J. and McPartland, J. (2005), "Understanding the nature of face processing impairment in autism: insights from behavioral and electrophysiological studies", Developmental Neuropsychology, Vol. 27 No. 3, pp. 403-424.

Dawson, G., Rogers, S., Munson, J., Smith, M., Winter, J. and Greenson, J. (2010), "Randomized, controlled trial of an intervention for toddlers with autism: the early start Denver model", PEDIATRICS, Vol. 125 No. 1, pp. 17-23.

Dawson, G., Webb, S.J., Carver, L., Panagiotides, H. and McPartland, J. (2004b), "Young children with autism show atypical brain responses to fearful versus neutral facial expressions of emotion", Developmental Science, Vol. 7 No. 3, pp. 340-359, doi: 10.1111/j.1467-7687. 2004.00352.x

De Vries, M. and Geurts, H.M. (2012), "Cognitive flexibility in ASD; task switching with emotional faces", Journal of Autism and Developmental Disorders, Vol. 42 No. 12, pp. 2558-2568.

Fabio, R.A. (2012), "Cognitive skills enhancement in intellectual disability", Intellectual Disability: Management, Causes and Social Perceptions, Vol. 33, pp. 147-166.

Fabio, R.A. and Caprì, T. (2019a), "Automatic and controlled attentional capture by threatening stimuli", Heliyon, Vol. 5, pp. 17-52, doi: 10.1016/j.heliyon.2019.e01752.

Fabio, R.A., Giannatiempo, S. and Caprì, T. (2019c), "Attention and identification of the same and the similar visual stimuli in Rett Syndrome", Life Span and Disabilities, Vol. XXII No. 1, pp. 113-127.

Fabio, R.A., Oliva, P. and Murdaca, A.M. (2011), "Systematic and emotional contents in overselectivity processes in autism", Research in Autism Spectrum Disorders, Vol. 5 No. 1, pp. 575 -583.

Fabio, R.A., Caprì, T., Lotan, M., Towey, G.E. and Martino, G. (2018a), "Motor abilities are related to the specific genotype in Rett Syndrome", in Urbano, K.V. (Ed.), Advances in Genetics Research, Vol. 18, Nova Science Publisher, New York, ISBN: 978-153613265-6;978-153613264-9.

Fabio, R.A., Caprì, T., Nucita, A., lannizzotto, G. and Mohammadhasani, N. (2018b), "Eye gaze digital games to improve motivational and attentional ability in Rett syndrome", Journal of Special Education and Rehabilitation, Vol. 19 Nos 3/4, pp. 105-126, doi: 10.19057/jser.2019.43.

Fabio, R.A., Gangemi, A., Caprì, T., Budden, S. and Falzone, A. (2018c), "Neurophysiological and cognitive effects of transcranial direct current stimulation in three girls with Rett Syndrome with chronic language impairments", Research in Developmental Disabilities, Vol. 76, pp. 76-87, doi: 10.1016/j.ridd.2018.03.008.

Fabio, R.A., Magaudda, C., Caprì, T., Towey, g. and Martino, G. (2018d), "Choice behavior in Rett Syndrome, the consistency parameter", Life Span and Disability, Vol. XXXI No. 1, pp. 47-62, doi: 10.1016/ j.ridd.2018.03.008.

Fabio, R.A., Caprì, T., Buzzai, C., Pittalà, V. and Gangemi, A. (2019b), "Auditory and visual oddball paradigm evaluated through P300 in five girls with Rett Syndrome", Neuroquantology, Vol. 17, pp. 40-49, doi: 10.14704/nq.2019.17.07.2591.

Fabio, R.A., Billeci, L., Crifaci, G., Troise, E., Tortorella, G. and Pioggia, G. (2016), "Cognitive training modifies frequency EEG bands and neuropsychological measures in Rett syndrome", Research in Developmental Disabilities, Vol. 54, pp. 73-85

Frye, D., Zelazo, P.D. and Palfai, T. (1995), "Theory of mind and rulebased reasoning", Cognitive Development, Vol. 10 No. 4, pp. 483-527.

Griffiths, R. (1984), The Abilities of Young Children, ARICD, Amersham.

Happe, F. and Frith, U. (2006), "The weak coherence account: detail-focused cognitive style in autism spectrum disorders", Journal of Autism and Developmental Disorders, Vol. 36 No. 1, pp. 5-25. 
Happé, F., Booth, R., Charlton, R. and Hughes, C. (2006), "Executive function deficits in autism spectrum disorders and attention-deficit/ hyperactivity disorder: examining profiles across domains and ages", Brain and Cognition, Vol. 61, pp. 25-39.

Kaland, N., Smith, L. and Mortensen, E.L. (2007), "Response times of children and adolescents with Asperger syndrome on an 'advanced' test of theory of mind", Journal of Autism and Developmental Disorders, Vol. 37 No. 2, pp. 197-209, doi: 10.1007/s10803-006-0152-8.

Klin, A., Jones, W., Schultz, R., Volkmar, F. and Cohen, D. (2002), "Visual fixation patterns during viewing of naturalistic social situations as predictors of social competence in individuals with autism", Archives of General Psychiatry, Vol. 59 No. 9, pp. 809-816.

Leo, M., Carcagnì, P., Distante, C., Mazzeo, P.L., Spagnolo, P., Levante, A. and Lecciso, F. (2019), "Computational analysis of deep visual data for quantifying facial expression production", Applied Sciences (Sciences, Vol. 9 No. 21, doi: 10.3390/app9214542.

Lord, C., Risi, S., Lambrecht, L., Cook, E.H., Jr., Leventhal, B.L. and DiLavore, P.C. (2000), "The autism diagnostic observation schedule-generic: a standard measure of social and communicative deficits associated with the spectrum of autism", Journal of Autism and Developmental Disorders, Vol. 30 No. 3, pp. 205-223.

Loth, E., Garrido, L., Ahmad, J., Watson, E., Duff, A. and Duchaine, B. (2018), "Facial expression recognition as a candidate marker for autism spectrum disorder: how frequent and severe are deficits?", Molecular Autism, Vol. 9 No. 1, p. 7.

Marchetti, A., Miraglia, L. and Di Dio, C. (2020), "Toward a socio-material approach to cognitive empathy in autistic spectrum disorder”, Frontiers in Psychology, Vol. 10, doi: 10.3389/fpsyg.2019.02965.

Russell, J. (1997), "How executive disorders can bring about an adequate theory of mind", in Russell J. (Ed.), Autism as an Executive Disorder, Oxford University Press: Oxford, pp. 256-304.

Van Eylen, L., Boets, B., Steyaert, J., Evers, K., Wagemans, J. and Noens, I. (2011), "Cognitive flexibility in autism spectrum disorder: explaining the inconsistencies?", Research in Autism Spectrum Disorders, Vol. 5 No. 4, pp. 1390-1401.

Weigelt, S., Koldewyn, K. and Kanwisher, N. (2013), "Face recognition deficits in autism spectrum disorders are both domain specific and process specific", PloS one, Vol. 8 No. 9, p. e74541.

Yerys, B.E., Wolff, B.C., Moody, E., Pennington, B.F. and Hepburn, S.L. (2012), "Brief report: impaired flexible item selection task (FIST) in school-age children with autism spectrum disorders", Journal of Autism and Developmental Disorders, Vol. 42 No. 9, pp. 2013-2020.

\section{Further reading}

Critchley, H.D., Daly, E.M., Bullmore, E.T., Williams, S.C.R., Van Amelsvoort, T. and Robertson, D.M. (2000), "The functional neuroanatomy of social behavior-changes in cerebral blood flow when people with autistic disorder process facial expressions", Brain, Vol. 123 No. 11, pp. 2203-2212.

Dawson, G., Toth, K., Abbott, R., Osterling, J., Munson, J. and Estes, A. (2004a), "Early social attention impairments in autism: social orienting, joint attention, and attention to distress", Developmental Psychology, Vol. 40 No. 2, pp. 271-283.

Fabio, R.A. and Urso, M. (2014), "The analysis of attention network in ADHD, attention problems and typically developing subjects", Life Span and Disability, Vol. 17 No. 2, pp. 199-221.

Geurts, H.M., Corbett, B. and Solomon, M. (2009), "The paradox of cognitive flexibility in autism", Trends in Cognitive Sciences, Vol. 13 No. 2, pp. 74-82.

Gross, T.F. (2004), "The perception of four basic emotions in human and nonhuman faces by children with autism and other developmental disabilities", Journal of Abnormal Child Psychology, Vol. 32 No. 5 , pp. 469-480, doi: 10.1023/B:JACP.0000037777.17698.01.

Gur, R.C., Sara, R., Hagendoorn, M., Marom, O., Hughett, P. and Macy, L. (2002), "A method for obtaining 3-dimensional facial expressions and its standardization for use in neurocognitive studies", Journal of Neuroscience Methods, Vol. 115 No. 2, pp. 137-143.

Hill, E.L. (2004), "Evaluating the theory of executive function deficits in autism", Developmental Review, Vol. 24 No. 2, pp. 189-233.

Hubl, D., Bolte, S., Feineis-Matthews, S., Lanfermann, H., Federspiel, A. and Strik, W. (2003), "Functional imbalance of visual pathways indicates alternative face processing strategies in autism", Neurology, Vol. 61 No. 9, pp. 1232-1237. 
Ivens, J. and Martin, N. (2022), "A common metric for the Griffiths scales", Archives of Disease in Childhood, Vol. 87 No. 2, pp. 109-110.

Korpilahti, P., Jansson-Verkasalo, E., Mattila, M.-L., Kuusikko, S., Suominen, K., Rytky, S., et al. (2007), "Processing of affective speech prosody is impaired in Asperger syndrome", Journal of Autism and Developmental Disorders, Vol. 37 No. 8, pp. 1539-1549, doi: 10.1007/s10803-006-0271-2.

Nelson, C.A. (2001), "The development and neural bases of face recognition", Infant and Child Development, Vol. 10 Nos 1/2, pp. 3-18.

Pierce, K., Muller, R.A., Ambrose, J., Allen, G. and Courchesne, E. (2001), "Face processing occurs outside the fusiform 'face area' in autism: evidence from functional MRl”, Brain, Vol. 124 No. 10, pp. 2059-2073.

Piggot, J., Kwon, H., Mobbs, D., Blasey, C., Lotspeich, L. and Menon, V. (2004), "Emotional attribution in high-functioning individuals with autistic spectrum disorder: a functional imaging study", Journal of the American Academy of Child \& Adolescent Psychiatry, Vol. 43 No. 4, pp. 473-480.

Russo, N., Flanagan, T., larocci, G., Berringer, D., Zelazo, P.D. and Burack, J.A. (2007), "Deconstructing executive deficits among persons with autism: implications for cognitive neuroscience", Brain and Cognition, Vol. 65 No. 1, pp. 77-86.

Schultz, R.T., Grelotti, D.J. and Pober, B.R. (2001), "Williams syndrome and brain-behavior relationships", Journal of the American Academy of Child \& Adolescent Psychiatry, Vol. 40 No. 5, pp. 606-609.

Schultz, R.T., Gauthier, I., Klin, A., Fulbright, R.K., Anderson, A.W. and Volkmar, F. (2000), "Abnormal ventral temporal cortical activity during face discrimination among individuals with autism and Asperger syndrome", Archives of General Psychiatry, Vol. 57 No. 4, pp. 331-340.

Uljarević, M. and Hamilton, A. (2013), "Recognition of emotions in autism: a formal meta-analysis", Journal of Autism and Developmental Disorders, Vol. 43, pp. 1517-1526.

Wang, A.T., Dapretto, M., Hariri, A.R., Sigman, M. and Bookheimer, S.Y. (2004), "Neural correlates of facial affect processing in children and adolescents with autism spectrum disorder", Journal of the American Academy of Child and Adolescent Psychiatry, Vol. 43 No. 4, pp. 481-490.

\section{Author affiliations}

Rosa Angela Fabio and Sonia Esposito are both based at the Department of Clinical and Experimental Medicine, University of Messina, Messina, Italy.

Cristina Carrozza is based at Research and Biomedical Innovation Institute, National Research Center, Messina, Italy.

Gaetana Pino is based at the Division of Child Neurology and Psychiatry, G. Martino Hospital, University of Messina, Messina, Italy.

Tindara Caprì is based at the Department of Clinical and Experimental Medicine, University of Messina, Messina, Italy.

\section{Corresponding author}

Tindara Caprì can be contacted at: tcapri@unime.it

For instructions on how to order reprints of this article, please visit our website: www.emeraldgrouppublishing.com/licensing/reprints.htm

Or contact us for further details: permissions@emeraldinsight.com 